\title{
New Families of Bi-Univalent Functions Governed by Gegenbauer Polynomials
}

\author{
Abbas Kareem Wanas \\ Department of Mathematics, College of Science, University of Al-Qadisiyah, Iraq \\ e-mail: abbas.kareem.w@qu.edu.iq
}

\begin{abstract}
The aim of this article is to initiating an exploration of the properties of bi-univalent functions related to Gegenbauer polynomials. To do so, we introduce a new families $\mathbb{T}_{\Sigma}(\gamma, \phi, \mu, \eta, \theta, \lambda, t, \delta)$ and $\mathbb{S}_{\Sigma}(\sigma, \eta, \theta, \lambda, t, \delta)$ of holomorphic and bi-univalent functions. We derive estimates on the initial coefficients and solve the Fekete-Szegö problem of functions in these families.
\end{abstract}

\section{Introduction}

"In [20] Legendre studied orthogonal polynomials comprehensively. The importance of orthogonal polynomials for contemporary mathematics as well as for a wide range of their applications in physics and engineering, is beyond any doubt. It is well-known that these polynomials play an essential role in problems of the approximation theory. They occur in the theory of differential and integral equations as well as in mathematical statistics. Their applications in quantum mechanics, scattering theory, automatic control, signal analysis, and axially symmetric potential theory are also known [7,12]. In practical, the Gegenbauer polynomials is special case of orthogonal polynomials. They are representatively related with typically real functions $T_{R}$ as discovered in [19]. Typically, real functions play an important role in the geometric function theory because of the relation $T_{R}=\overline{c o} S_{R}$ and its role of estimating coefficient bounds, where $S_{R}$ indicates the family of univalent functions in the unit disk with real coefficients and $\overline{c o} S_{R}$ denotes the closed convex hull of $S_{R}$."

Received: September 6, 2021; Accepted: September 25, 2021

2010 Mathematics Subject Classification: 30C45, 30C50.

Keywords and phrases: holomorphic function, bi-univalent function, Gegenbauer polynomials, Fekete-Szegö problem, coefficient estimates. 
On this subject in geometric function theory, the so-called Fekete-Szegö type inequalities (or problems) which estimate some upper bounds for $\left|a_{3}-\mu a_{2}^{2}\right|$ for holomorphic univalent functions. Its origin was in the disproof by Fekete and Szegö [16] conjecture of Littlewood and Paley that the coefficients of odd univalent functions are bounded by unity.

Let $\mathcal{A}$ stand for the collection of functions $f$ have the type:

$$
f(z)=z+\sum_{n=2}^{\infty} a_{n} z^{n},
$$

which are holomorphic in the open unit disk $U=\{z \in \mathbb{C}:|z|<1\}$.

Further, symbolized by $S$ the subfamily of $\mathcal{A}$ consisting the functions that are univalent in $U$.

According to the "Koebe One-Quarter Theorem" [13] each function $f$ from $S$ has an inverse $f^{-1}$, which fulfills

$$
f^{-1}(f(z))=z, \quad(z \in U)
$$

and

$$
f\left(f^{-1}(w)\right)=w, \quad\left(|w|<r_{0}(f), r_{0}(f) \geq \frac{1}{4}\right)
$$

where

$$
g(w)=f^{-1}(w)=w-a_{2} w^{2}+\left(2 a_{2}^{2}-a_{3}\right) w^{3}-\left(5 a_{2}^{3}-5 a_{2} a_{3}+a_{4}\right) w^{4}+\cdots .
$$

A function $f \in \mathcal{A}$ is named bi-univalent in $U$ if together $f$ and $f^{-1}$ are univalent in $U$. Let $\Sigma$ indicate the family of bi-univalent functions in $U$ satisfying (1.1). Beginning with Srivastava et al. pioneering work [36] on the subject, the large number of works associated with the subject have been presented (see, for example [1,2,4,5,8,9,10,11,14, $17,18,21,22,25,28,29,30,31,32,33,34,35,37,38,39,40,41])$. We see that the set $\Sigma$ is not empty. We see that the functions

$$
\frac{z}{1-z}, \frac{1}{2} \log \left(\frac{1+z}{1-z}\right) \text { and }-\log (1-z)
$$

are in the set $\Sigma$ with the corresponding inverse functions

$$
\frac{w}{1+w}, \frac{e^{2 w}-1}{e^{2 w}+1} \text { and } \frac{e^{w}-1}{e^{w}},
$$


respectively. But the functions

$$
z-\frac{z^{2}}{2} \text { and } \frac{z}{1-z^{2}}
$$

are not a member of the set $\Sigma$.

The problem to find the bound of $\left|a_{n}\right|,(n=3,4, \ldots)$ of functions $f \in \Sigma$ is still an open problem.

The fundamental distributions like, the Pascal, the Binomial, the Poisson, the Logarithmic, the Borel have been partially considered in the "Geometric Function Theory" from a theoretical point of view (see for example $[6,15,24,26,43])$.

We say that the discrete random variable $x$ have a beta negative binomial distribution, if it has the values $0,1,2,3, \ldots$ with the probabilities $\frac{\beta(\eta+\theta, \lambda)}{\beta(\eta, \lambda)}, \theta \frac{\beta(\eta+\theta, \lambda+1)}{\beta(\eta, \lambda)}, \frac{1}{2} \theta(\theta+$ 1) $\frac{\beta(\eta+\theta, \lambda+2)}{\beta(\eta, \lambda)}, \ldots$, respectively, where $\eta, \theta, \lambda$ are named the parameters.

$$
\begin{aligned}
\operatorname{Prob}(x=\tau) & =\left(\begin{array}{c}
\theta+\tau-1 \\
\tau
\end{array}\right) \frac{\beta(\eta+\theta, \lambda+\tau)}{\beta(\eta, \lambda)}=\frac{\Gamma(\theta+\tau)}{\tau ! \Gamma(\theta)} \frac{\Gamma(\eta+\theta) \Gamma(\lambda+\tau) \Gamma(\eta+\lambda)}{\Gamma(\eta+\theta+\lambda+\tau) \Gamma(\eta) \Gamma(\lambda)} \\
& =\frac{(\eta)_{\theta}(\theta)_{\tau}(\lambda)_{\tau}}{(\eta+\lambda)_{\theta}(\theta+\eta+\lambda)_{\tau} \tau !}, \tau=0,1,2,3, \ldots,
\end{aligned}
$$

where $(\alpha)_{n}$ is the Pochhammer symbol defined by

$$
(\alpha)_{n}=\frac{\Gamma(\alpha+n)}{\Gamma(\alpha)}= \begin{cases}1 & (n=0) \\ \alpha(\alpha+1) \ldots(\alpha+n-1) & (n \in \mathbb{N}) .\end{cases}
$$

Recently, Wanas and Al-Ziadi [42] studied the following power series whose coefficients are probabilities of the beta negative binomial distribution:

$$
\mathfrak{X}_{\eta, \lambda}^{\theta}(z)=z+\sum_{n=2}^{\infty} \frac{(\eta)_{\theta}(\theta)_{n-1}(\lambda)_{n-1}}{(\eta+\lambda)_{\theta}(\theta+\eta+\lambda)_{n-1}(n-1) !} z^{n}, \quad z \in U,
$$

where $\eta, \lambda, \theta>0$. We see that, by making use of ratio test we deduce that the radius of convergence of the above power series is infinity.

Now, we consider the linear operator $\mathfrak{B}_{\eta, \lambda}^{\theta}: \mathcal{A} \rightarrow \mathcal{A}$ which is defined as follows:

$$
\mathfrak{B}_{\eta, \lambda}^{\theta} f(z)=\mathfrak{X}_{\eta, \lambda}^{\theta}(z) * f(z)=z+\sum_{n=2}^{\infty} \frac{(\eta)_{\theta}(\theta)_{n-1}(\lambda)_{n-1}}{(\eta+\lambda)_{\theta}(\theta+\eta+\lambda)_{n-1}(n-1) !} a_{n} z^{n}, \quad z \in U,
$$


where " $*$ " indicate the convolution of two series.

"For the functions $f$ and $g$ be holomorphic in $U$. We say that the function $f$ is said to be subordinate to $g$, if there exists a Schwarz function $w$ holomorphic in $U$ with $w(0)=$ 0 and $|w(z)|<1(z \in U)$ such that $f(z)=g(w(z))$. This subordination is indicated by $f \prec g$ or $f(z) \prec g(z)(z \in U)$. It is well known that (see [23]), if the function $g$ is univalent in $U$, then $f \prec g$ if and only if $f(0)=g(0)$ and $f(U) \subset g(U)$."

Recently, Amourah [3] studied the generating function of Gegenbauer polynomials $H_{\delta}(z, t)$ that are given by the following recurrence relation:

$$
H_{\delta}(z, t)=\frac{1}{\left(1-2 t z+z^{2}\right)^{\delta}}
$$

where $\delta \in \mathbb{R} \backslash\{0\}, t \in[-1,1]$ and $z \in U$. For fixed $t$, the function $H_{\delta}$ is holomorphic in $U$, so it may be expanded in a Taylor-Maclaurin series as note that if $t=\cos \beta$, where $\beta \in\left(-\frac{\pi}{3}, \frac{\pi}{3}\right)$, then

$$
H_{\delta}(z, t)=\frac{1}{\left(1-2 t z+z^{2}\right)^{\delta}}=\sum_{n=0}^{\infty} \mathcal{G}_{n}^{\delta}(t) z^{n},
$$

where $\mathcal{G}_{n}^{\delta}(t)$ is Gegenbauer polynomial of degree $n$.

Clearly, $H_{\delta}$ generates nothing when $\delta=0$. Thus, the generating function of the Gegenbauer polynomial is set to be

$$
H_{0}(z, t)=1-\log \left(1-2 t z+z^{2}\right)=\sum_{n=0}^{\infty} \mathcal{G}_{n}^{0}(t) z^{n} .
$$

Furthermore, it is worth to mention that a normalization of $\delta$ to be greater than $-\frac{1}{2}$ is desirable [12,27]. Also, Gegenbauer polynomials can be introduced by the following recurrence relations:

$$
\mathcal{G}_{n}^{\delta}(t)=\frac{1}{2}\left[2 t(n+\delta-1) \mathcal{G}_{n-1}^{\delta}(t)-(n+2 \delta-2) \mathcal{G}_{n-1}^{\delta}(t)\right]
$$

with the initial values

$$
\mathcal{G}_{0}^{\delta}(t)=1, \mathcal{G}_{1}^{\delta}(t)=2 \delta t \text { and } \mathcal{G}_{2}^{\delta}(t)=2 \delta(\delta+1) t^{2}-\delta .
$$

Remark 1.1. Choosing the special values of $\delta$, the Gegenbauer polynomial $\mathcal{G}_{n}^{\delta}(t)$ reduces to the following well-known polynomials: 
1) Taking $\delta=1$, we have the Chebyshev Polynomials.

2) Taking $\delta=\frac{1}{2}$, we obtain the Legendre Polynomials.

\section{Main Results}

This section start with defining the families $\mathbb{T}_{\Sigma}(\gamma, \phi, \mu, \eta, \theta, \lambda, t, \delta)$ and $\mathbb{S}_{\Sigma}(\sigma, \eta, \theta, \lambda, t, \delta)$ as follows:

Definition 2.1. For $0 \leq \gamma \leq 1,0 \leq \phi \leq 1,0 \leq \mu \leq 1, t \in\left(\frac{1}{2}, 1\right]$ and $\delta$ is a nonzero real constant, a function $f \in \Sigma$ is called in the family $\mathbb{T}_{\Sigma}(\gamma, \phi, \mu, \eta, \theta, \lambda, t, \delta)$ if it fulfills the subordinations:

$$
\left(\frac{z\left(\mathfrak{B}_{\eta, \lambda}^{\theta} f(z)\right)^{\prime}}{\mathfrak{B}_{\eta, \lambda}^{\theta} f(z)}\right)^{\gamma}\left[(1-\mu) \frac{z\left(\mathfrak{B}_{\eta, \lambda}^{\theta} f(z)\right)^{\prime}}{\mathfrak{B}_{\eta, \lambda}^{\theta} f(z)}+\mu\left(1+\frac{z\left(\mathfrak{B}_{\eta, \lambda}^{\theta} f(z)\right)^{\prime \prime}}{\left(\mathfrak{B}_{\eta, \lambda}^{\theta} f(z)\right)^{\prime}}\right)\right]^{\phi} \prec \frac{1}{\left(1-2 t z+z^{2}\right)^{\delta}}
$$

and

$$
\left(\frac{w\left(\mathfrak{B}_{\eta, \lambda}^{\theta} g(w)\right)^{\prime}}{\mathfrak{B}_{\eta, \lambda}^{\theta} g(w)}\right)^{\gamma}\left[(1-\mu) \frac{w\left(\mathfrak{B}_{\eta, \lambda}^{\theta} g(w)\right)^{\prime}}{\mathfrak{B}_{\eta, \lambda}^{\theta} g(w)}+\mu\left(1+\frac{w\left(\mathfrak{B}_{\eta, \lambda}^{\theta} g(w)\right)^{\prime \prime}}{\left(\mathfrak{B}_{\eta, \lambda}^{\theta} g(w)\right)^{\prime}}\right)\right]^{\phi}<\frac{1}{\left(1-2 t w+w^{2}\right)^{\delta}}
$$

where the function $g=f^{-1}$ is given by (1.2).

Definition 2.2. For $0 \leq \sigma \leq 1, t \in\left(\frac{1}{2}, 1\right]$ and $\delta$ is a nonzero real constant, a function $f \in \Sigma$ is called in the family $\mathbb{S}_{\Sigma}(\sigma, \eta, \theta, \lambda, t, \delta)$ if it fulfills the subordinations:

$$
\frac{z\left(\mathfrak{B}_{\eta, \lambda}^{\theta} f(z)\right)^{\prime}+(2 \sigma+1) z^{2}\left(\mathfrak{B}_{\eta, \mathrm{A}}^{\theta} f(z)\right)^{\prime \prime}+\sigma z^{3}\left(\mathfrak{B}_{\eta, \mathrm{A}}^{\theta} f(z)\right)^{\prime \prime \prime}}{z\left(\mathfrak{B}_{\eta, \mathrm{A}}^{\theta} f(z)\right)^{\prime}+\sigma z^{2}\left(\mathfrak{B}_{\eta, \lambda}^{\theta} f(z)\right)^{\prime \prime}} \prec \frac{1}{\left(1-2 t z+z^{2}\right)^{\delta}}
$$

and

$$
\frac{w\left(\mathfrak{B}_{\eta, \lambda}^{\theta} g(w)\right)^{\prime}+(2 \sigma+1) w^{2}\left(\mathfrak{B}_{\eta, \lambda}^{\theta} g(w)\right)^{\prime \prime}+\sigma w^{3}\left(\mathfrak{B}_{\eta, \lambda}^{\theta} g(w)\right)^{\prime \prime \prime}}{w\left(\mathfrak{B}_{\eta, \lambda}^{\theta} g(w)\right)^{\prime}+\sigma w^{2}\left(\mathfrak{B}_{\eta, \lambda}^{\theta} g(w)\right)^{\prime \prime}}<\frac{1}{\left(1-2 t w+w^{2}\right)^{\delta}},
$$

where the function $g=f^{-1}$ is given by (1.2).

In particular, if we choose $\phi=0$ and $\gamma=1$ in Definition 2.1 , the family $\mathbb{T}_{\Sigma}(\gamma, \phi, \mu, \eta, \theta, \lambda, t, \delta)$ reduces to the family $\mathfrak{I}_{\Sigma}(\eta, \theta, \lambda, t, \delta)$ of bi-starlike functions 
which fulfills the conditions:

$$
\frac{z\left(\mathfrak{B}_{\eta, \lambda}^{\theta} f(z)\right)^{\prime}}{\mathfrak{B}_{\eta, \lambda}^{\theta} f(z)} \prec \frac{1}{\left(1-2 t z+z^{2}\right)^{\delta}}
$$

and

$$
\frac{w\left(\mathfrak{B}_{\eta, \lambda}^{\theta} g(w)\right)^{\prime}}{\mathfrak{B}_{\eta, \lambda}^{\theta} g(w)} \prec \frac{1}{\left(1-2 t w+w^{2}\right)^{\delta}},
$$

where the function $g=f^{-1}$ is given by (1.2).

If we choose $\sigma=0$ in Definition 2.2, the family $\mathbb{S}_{\Sigma}(\sigma, \eta, \theta, \lambda, t, \delta)$ reduces to the family $\mathfrak{G}_{\Sigma}(\eta, \theta, \lambda, t, \delta)$ of bi-convex functions which fulfills the conditions:

$$
1+\frac{z\left(\mathfrak{B}_{\eta, \lambda}^{\theta} f(z)\right)^{\prime \prime}}{\left(\mathfrak{B}_{\eta, \lambda}^{\theta} f(z)\right)^{\prime}} \prec \frac{1}{\left(1-2 t z+z^{2}\right)^{\delta}}
$$

and

$$
1+\frac{w\left(\mathfrak{B}_{\eta, \lambda}^{\theta} g(w)\right)^{\prime \prime}}{\left(\mathfrak{B}_{\eta, \lambda}^{\theta} g(w)\right)^{\prime}} \prec \frac{1}{\left(1-2 t w+w^{2}\right)^{\delta}},
$$

where the function $g=f^{-1}$ is given by (1.2).

Theorem 2.1. For $0 \leq \gamma \leq 1,0 \leq \phi \leq 1,0 \leq \mu \leq 1, t \in\left(\frac{1}{2}, 1\right]$ and $\delta$ is a nonzero real constant, let $f \in \mathcal{A}$ be in the family $\mathbb{T}_{\Sigma}(\gamma, \phi, \mu, \eta, \theta, \lambda, t, \delta)$. Then

$$
\left|a_{2}\right| \leq \frac{2|\delta| t \Gamma(\eta+\theta+\lambda+1) \Gamma(\eta) \Gamma(\lambda) \sqrt{2|\delta| t}}{\sqrt{\delta \theta^{2}(\gamma+\phi(\mu+1))^{2} \Gamma^{2}(\eta+\theta) \Gamma^{2}(\lambda+1) \Gamma^{2}(\eta+\lambda)}}
$$

and

$$
\left|a_{3}\right| \leq \frac{4 \Gamma^{2}(\eta+\theta+\lambda+1) \Gamma^{2}(\eta) \Gamma^{2}(\lambda) \delta^{2} t^{2}}{\theta^{2}(\gamma+\phi(\mu+1))^{2} \Gamma^{2}(\eta+\theta) \Gamma^{2}(\lambda+1) \Gamma^{2}(\eta+\lambda)}
$$




$$
+\frac{2 \Gamma(\eta+\theta+\lambda+2) \Gamma(\eta) \Gamma(\lambda)|\delta| t}{\theta(\theta+1)(\gamma+\phi(2 \mu+1)) \Gamma(\eta+\theta) \Gamma(\lambda+2) \Gamma(\eta+\lambda)},
$$

where

$$
\begin{aligned}
& \Omega(\gamma, \phi, \mu, \eta, \theta, \lambda)=\frac{2(\theta+1)(\gamma+\phi(2 \mu+1)) \Gamma(\lambda+2)}{\Gamma(\eta+\theta+\lambda+2)} \\
& +\frac{\theta \Gamma(\eta+\theta) \Gamma^{2}(\lambda+1) \Gamma(\eta+\lambda)[\gamma(\gamma-1)+\phi(\mu+1)(2 \gamma+(\phi-1)(\mu+1))-2(\gamma+\phi(3 \mu+1))]}{\Gamma^{2}(\eta+\theta+\lambda+1) \Gamma(\eta) \Gamma(\lambda)} .
\end{aligned}
$$

Proof. Let $f \in \mathbb{T}_{\Sigma}(\gamma, \phi, \mu, \eta, \theta, \lambda, t, \delta)$. Then there exists two holomorphic functions $u, v: U \rightarrow U$ given by

$$
u(z)=u_{1} z+u_{2} z^{2}+u_{3} z^{3}+\cdots \quad(z \in U)
$$

and

$$
v(w)=v_{1} w+v_{2} w^{2}+v_{3} w^{3}+\cdots \quad(w \in U),
$$

with $u(0)=v(0)=0,|u(z)|<1,|v(w)|<1, z, w \in U$ such that

$$
\begin{aligned}
& \left(\frac{z\left(\mathfrak{B}_{\eta, \lambda}^{\theta} f(z)\right)^{\prime}}{\mathfrak{B}_{\eta, \lambda}^{\theta} f(z)}\right)^{\gamma}\left[(1-\mu) \frac{z\left(\mathfrak{B}_{\eta, \lambda}^{\theta} f(z)\right)^{\prime}}{\mathfrak{B}_{\eta, \lambda}^{\theta} f(z)}+\mu\left(1+\frac{z\left(\mathfrak{B}_{\eta, \lambda}^{\theta} f(z)\right)^{\prime \prime}}{\left(\mathfrak{B}_{\eta, \lambda}^{\theta} f(z)\right)^{\prime}}\right)\right]^{\phi} \\
= & 1+\mathcal{G}_{1}^{\delta}(t) u(z)+\mathcal{G}_{2}^{\delta}(t) u^{2}(z)+\cdots
\end{aligned}
$$

and

$$
\begin{aligned}
& \left(\frac{w\left(\mathfrak{B}_{\eta, \lambda}^{\theta} g(w)\right)^{\prime}}{\mathfrak{B}_{\eta, \lambda}^{\theta} g(w)}\right)^{\gamma}\left[(1-\mu) \frac{w\left(\mathfrak{B}_{\eta, \lambda}^{\theta} g(w)\right)^{\prime}}{\mathfrak{B}_{\eta, \lambda}^{\theta} g(w)}+\mu\left(1+\frac{w\left(\mathfrak{B}_{\eta, \lambda}^{\theta} g(w)\right)^{\prime \prime}}{\left(\mathfrak{B}_{\eta, \lambda}^{\theta} g(w)\right)^{\prime}}\right)\right]^{\phi} \\
= & 1+\mathcal{G}_{1}^{\delta}(t) v(w)+\mathcal{G}_{2}^{\delta}(t) v^{2}(w)+\cdots .
\end{aligned}
$$

Combining (2.2), (2.3), (2.4) and (2.5), we obtain

$$
\begin{aligned}
& \left(\frac{z\left(\mathfrak{B}_{\eta, \lambda}^{\theta} f(z)\right)^{\prime}}{\mathfrak{B}_{\eta, \lambda}^{\theta} f(z)}\right)^{\gamma}\left[(1-\mu) \frac{z\left(\mathfrak{B}_{\eta, \lambda}^{\theta} f(z)\right)^{\prime}}{\mathfrak{B}_{\eta, \lambda}^{\theta} f(z)}+\mu\left(1+\frac{z\left(\mathfrak{B}_{\eta, \lambda}^{\theta} f(z)\right)^{\prime \prime}}{\left(\mathfrak{B}_{\eta, \lambda}^{\theta} f(z)\right)^{\prime}}\right)\right]^{\phi} \\
= & 1+\mathcal{G}_{1}^{\delta}(t) u_{1} z+\left[\mathcal{G}_{1}^{\delta}(t) u_{2}+\mathcal{G}_{2}^{\delta}(t) u_{1}^{2}\right] z^{2}+\cdots
\end{aligned}
$$


and

$$
\begin{aligned}
& \left(\frac{w\left(\mathfrak{B}_{\eta, \lambda}^{\theta} g(w)\right)^{\prime}}{\mathfrak{B}_{\eta, \lambda}^{\theta} g(w)}\right)^{\gamma}\left[(1-\mu) \frac{w\left(\mathfrak{B}_{\eta, \lambda}^{\theta} g(w)\right)^{\prime}}{\mathfrak{B}_{\eta, \lambda}^{\theta} g(w)}+\mu\left(1+\frac{w\left(\mathfrak{B}_{\eta, \lambda}^{\theta} g(w)\right)^{\prime \prime}}{\left(\mathfrak{B}_{\eta, \lambda}^{\theta} g(w)\right)^{\prime}}\right)\right]^{\phi} \\
= & 1+\mathcal{G}_{1}^{\delta}(t) v_{1} w+\left[\mathcal{G}_{1}^{\delta}(t) v_{2}+\mathcal{G}_{2}^{\delta}(t) v_{1}^{2}\right] w^{2}+\cdots .
\end{aligned}
$$

It is quite well-known that if $|u(z)|<1$ and $|v(w)|<1, z, w \in U$, then

$$
\left|u_{i}\right| \leq 1 \quad \text { and } \quad\left|v_{i}\right| \leq 1 \quad \text { for all } i \in \mathbb{N} \text {. }
$$

Equating the coefficients in (2.6) and (2.7), we deduce that

$$
\frac{\theta(\gamma+\phi(\mu+1)) \Gamma(\eta+\theta) \Gamma(\lambda+1) \Gamma(\eta+\lambda)}{\Gamma(\eta+\theta+\lambda+1) \Gamma(\eta) \Gamma(\lambda)} a_{2}=\mathcal{G}_{1}^{\delta}(t) u_{1}
$$

$$
\frac{\theta(\theta+1)(\gamma+\phi(2 \mu+1)) \Gamma(\eta+\theta) \Gamma(\lambda+2) \Gamma(\eta+\lambda)}{\Gamma(\eta+\theta+\lambda+2) \Gamma(\eta) \Gamma(\lambda)} a_{3}
$$$$
+\frac{\theta^{2} \Gamma^{2}(\eta+\theta) \Gamma^{2}(\lambda+1) \Gamma^{2}(\eta+\lambda)[\gamma(\gamma-1)+\phi(\mu+1)(2 \gamma+(\phi-1)(\mu+1))-2(\gamma+\phi(3 \mu+1))]}{2 \Gamma^{2}(\eta+\theta+\lambda+1) \Gamma^{2}(\eta) \Gamma^{2}(\lambda)} a_{2}^{2}
$$$$
=\mathcal{G}_{1}^{\delta}(t) u_{2}+\mathcal{G}_{2}^{\delta}(t) u_{1}^{2},
$$

$$
-\frac{\theta(\gamma+\phi(\mu+1)) \Gamma(\eta+\theta) \Gamma(\lambda+1) \Gamma(\eta+\lambda)}{\Gamma(\eta+\theta+\lambda+1) \Gamma(\eta) \Gamma(\lambda)} a_{2}=\mathcal{G}_{1}^{\delta}(t) v_{1}
$$

and

$\frac{\theta(\theta+1)(\gamma+\phi(2 \mu+1)) \Gamma(\eta+\theta) \Gamma(\lambda+2) \Gamma(\eta+\lambda)}{\Gamma(\eta+\theta+\lambda+2) \Gamma(\eta) \Gamma(\lambda)}\left(2 a_{2}^{2}-a_{3}\right)$

$+\frac{\theta^{2} \Gamma^{2}(\eta+\theta) \Gamma^{2}(\lambda+1) \Gamma^{2}(\eta+\lambda)[\gamma(\gamma-1)+\phi(\mu+1)(2 \gamma+(\phi-1)(\mu+1))-2(\gamma+\phi(3 \mu+1))]}{2 \Gamma^{2}(\eta+\theta+\lambda+1) \Gamma^{2}(\eta) \Gamma^{2}(\lambda)} a_{2}^{2}$

$=\mathcal{G}_{1}^{\delta}(t) v_{2}+\mathcal{G}_{2}^{\delta}(t) v_{1}^{2}$.

From (2.9) and (2.11), we conclude that

$$
u_{1}=-v_{1}
$$

and

$$
\frac{2 \theta^{2}(\gamma+\phi(\mu+1))^{2} \Gamma^{2}(\eta+\theta) \Gamma^{2}(\lambda+1) \Gamma^{2}(\eta+\lambda)}{\Gamma^{2}(\eta+\theta+\lambda+1) \Gamma^{2}(\eta) \Gamma^{2}(\lambda)} a_{2}^{2}=\left(\mathcal{G}_{1}^{\delta}(t)\right)^{2}\left(u_{1}^{2}+v_{1}^{2}\right) \text {. }
$$


Adding (2.10) to (2.12), yields

$$
\frac{\theta \Gamma(\eta+\theta) \Gamma(\eta+\lambda)}{\Gamma(\eta) \Gamma(\lambda)} \Omega(\gamma, \phi, \mu) a_{2}^{2}=\mathcal{G}_{1}^{\delta}(t)\left(u_{2}+v_{2}\right)+\mathcal{G}_{2}^{\delta}(t)\left(u_{1}^{2}+v_{1}^{2}\right),
$$

where $\Omega(\gamma, \phi, \mu, \eta, \theta, \lambda)$ is given by (2.1). Consequently, we have

$$
\begin{aligned}
& {\left[\frac{\theta \Gamma(\eta+\theta) \Gamma(\eta+\lambda)}{\Gamma(\eta) \Gamma(\lambda)} \Omega(\gamma, \phi, \mu)-\frac{2 \theta^{2}(\gamma+\phi(\mu+1))^{2} \Gamma^{2}(\eta+\theta) \Gamma^{2}(\lambda+1) \Gamma^{2}(\eta+\lambda) \mathcal{G}_{2}^{\delta}(t)}{\Gamma^{2}(\eta+\theta+\lambda+1) \Gamma^{2}(\eta) \Gamma^{2}(\lambda)\left(\mathcal{G}_{1}^{\delta}(t)\right)^{2}}\right] a_{2}^{2}} \\
& =\mathcal{G}_{1}^{\delta}(t)\left(u_{2}+v_{2}\right) .
\end{aligned}
$$

Further computations using (1.3), (2.8) and (2.15), we obtain

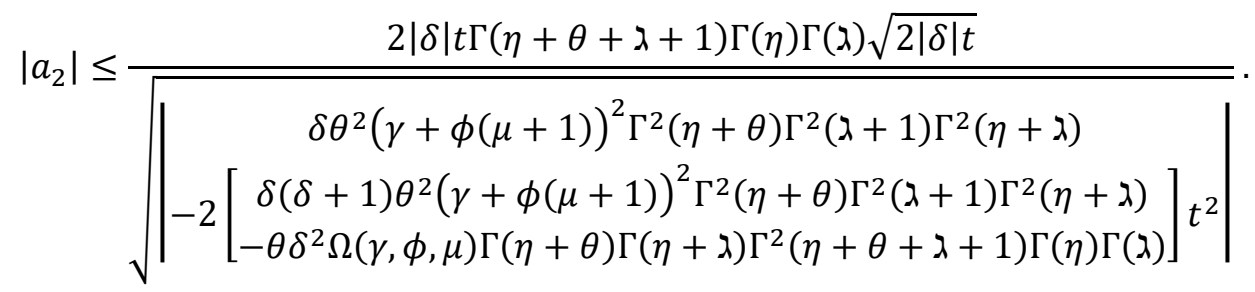

Next, if we subtract (2.12) from (2.10), we deduce that

$$
\begin{aligned}
& \frac{2 \theta(\theta+1)(\gamma+\phi(2 \mu+1)) \Gamma(\eta+\theta) \Gamma(\lambda+2) \Gamma(\eta+\lambda)}{\Gamma(\eta+\theta+\lambda+2) \Gamma(\eta) \Gamma(\lambda)}\left(a_{3}-a_{2}^{2}\right) \\
= & \mathcal{G}_{1}^{\delta}(t)\left(u_{2}-v_{2}\right)+\mathcal{G}_{2}^{\delta}(t)\left(u_{1}^{2}-v_{1}^{2}\right) .
\end{aligned}
$$

In view of (2.13) and (2.14), we get from (2.16)

$$
\begin{gathered}
a_{3}=\frac{\Gamma^{2}(\eta+\theta+\lambda+1) \Gamma^{2}(\eta) \Gamma^{2}(\lambda)\left(\mathcal{G}_{1}^{\delta}(t)\right)^{2}}{2 \theta^{2}(\gamma+\phi(\mu+1))^{2} \Gamma^{2}(\eta+\theta) \Gamma^{2}(\lambda+1) \Gamma^{2}(\eta+\lambda)}\left(u_{1}^{2}+v_{1}^{2}\right) \\
+\frac{\Gamma(\eta+\theta+\lambda+2) \Gamma(\eta) \Gamma(\lambda) \mathcal{G}_{1}^{\delta}(t)}{2 \theta(\theta+1)(\gamma+\phi(2 \mu+1)) \Gamma(\eta+\theta) \Gamma(\lambda+2) \Gamma(\eta+\lambda)}\left(u_{2}-v_{2}\right) .
\end{gathered}
$$

Thus applying (1.3), we obtain

$$
\begin{aligned}
\left|a_{3}\right| \leq \frac{4 \Gamma^{2}(\eta+\theta+\lambda+1) \Gamma^{2}(\eta) \Gamma^{2}(\lambda) \delta^{2} t^{2}}{\theta^{2}(\gamma+\phi(\mu+1))^{2} \Gamma^{2}(\eta+\theta) \Gamma^{2}(\lambda+1) \Gamma^{2}(\eta+\lambda)} \\
+\frac{2 \Gamma(\eta+\theta+\lambda+2) \Gamma(\eta) \Gamma(\lambda)|\delta| t}{\theta(\theta+1)(\gamma+\phi(2 \mu+1)) \Gamma(\eta+\theta) \Gamma(\lambda+2) \Gamma(\eta+\lambda)} .
\end{aligned}
$$


Putting $\phi=0$ and $\gamma=1$ in Theorem 2.1, we demonstrate the next outcome:

Corollary 2.1. For $t \in\left(\frac{1}{2}, 1\right]$ and $\delta$ is a nonzero real constant, let $f \in \mathcal{A}$ be in the family $\mathfrak{I}_{\Sigma}(\eta, \theta, \lambda, t, \delta)$. Then

$$
\left|a_{2}\right| \leq \frac{2|\delta| t \Gamma(\eta+\theta+\lambda+1) \Gamma(\eta) \Gamma(\lambda) \sqrt{2|\delta| t}}{\sqrt{\delta \theta^{2} \Gamma^{2}(\eta+\theta) \Gamma^{2}(\lambda+1) \Gamma^{2}(\eta+\lambda)}}
$$

and

$$
\left|a_{3}\right| \leq \frac{4 \Gamma^{2}(\eta+\theta+\lambda+1) \Gamma^{2}(\eta) \Gamma^{2}(\lambda) \delta^{2} t^{2}}{\theta^{2} \Gamma^{2}(\eta+\theta) \Gamma^{2}(\lambda+1) \Gamma^{2}(\eta+\lambda)}+\frac{2 \Gamma(\eta+\theta+\lambda+2) \Gamma(\eta) \Gamma(\lambda)|\delta| t}{\theta(\theta+1) \Gamma(\eta+\theta) \Gamma(\lambda+2) \Gamma(\eta+\lambda)},
$$

where

$$
\Im(\eta, \theta, \lambda)=\frac{2(\theta+1) \Gamma(\lambda+2)}{\Gamma(\eta+\theta+\lambda+2)}+\frac{-2 \theta \Gamma(\eta+\theta) \Gamma^{2}(\lambda+1) \Gamma(\eta+\lambda)}{\Gamma^{2}(\eta+\theta+\lambda+1) \Gamma(\eta) \Gamma(\lambda)} .
$$

Theorem 2.2. For $0 \leq \sigma \leq 1, t \in\left(\frac{1}{2}, 1\right]$ and $\delta$ is a nonzero real constant, let $f \in \mathcal{A}$ be in the family $\mathbb{S}_{\Sigma}(\sigma, \eta, \theta, \lambda, t, \delta)$. Then

$$
\left|a_{2}\right| \leq \frac{\Gamma(\eta+\theta+\lambda+1) \Gamma(\eta) \Gamma(\lambda)|\delta| t \sqrt{|\delta| t}}{\sqrt{\delta \theta^{2}(\sigma+1)^{2} \Gamma^{2}(\eta+\theta) \Gamma^{2}(\lambda+1) \Gamma^{2}(\eta+\lambda)} \begin{array}{c}
2 \delta(\delta+1) \theta^{2}(\sigma+1)^{2} \Gamma^{2}(\eta+\theta) \Gamma^{2}(\lambda+1) \Gamma^{2}(\eta+\lambda) \\
-\left[\begin{array}{c}
2 \delta \\
-\theta \delta^{2} \Upsilon(\sigma, \eta, \theta, \lambda) \Gamma(\eta+\theta) \Gamma(\eta+\lambda) \Gamma^{2}(\eta+\theta+\lambda+1) \Gamma(\eta) \Gamma(\lambda)
\end{array}\right] t^{2}
\end{array} \mid}
$$

and

$$
\begin{aligned}
\left|a_{3}\right| \leq \frac{\Gamma^{2}(\eta+\theta+\lambda+1) \Gamma^{2}(\eta) \Gamma^{2}(\lambda) \delta^{2} t^{2}}{\theta^{2}(\sigma+1)^{2} \Gamma^{2}(\eta+\theta) \Gamma^{2}(\lambda+1) \Gamma^{2}(\eta+\lambda)} \\
+\frac{2 \Gamma(\eta+\theta+\lambda+2) \Gamma(\eta) \Gamma(\lambda)|\delta| t}{3 \theta(\theta+1)(2 \sigma+1) \Gamma(\eta+\theta) \Gamma(\lambda+2) \Gamma(\eta+\lambda)}
\end{aligned}
$$

where

$$
\Upsilon(\sigma, \eta, \theta, \lambda)=\frac{3(\theta+1)(2 \sigma+1) \Gamma(\lambda+2)}{\Gamma(\eta+\theta+\lambda+2)}
$$




$$
-\frac{4 \theta(\sigma+1)^{2} \Gamma(\eta+\theta) \Gamma^{2}(\lambda+1) \Gamma(\eta+\lambda)}{\Gamma^{2}(\eta+\theta+\lambda+1) \Gamma(\eta) \Gamma(\lambda)} .
$$

Proof. Let $f \in \mathbb{S}_{\Sigma}(\sigma, \eta, \theta, \lambda, t, \delta)$. Then there exist two holomorphic functions $u, v: U \rightarrow U$

$$
\begin{aligned}
& \frac{z\left(\mathfrak{B}_{\eta, \lambda}^{\theta} f(z)\right)^{\prime}+(2 \sigma+1) z^{2}\left(\mathfrak{B}_{\eta, \lambda}^{\theta} f(z)\right)^{\prime \prime}+\sigma z^{3}\left(\mathfrak{B}_{\eta, \lambda}^{\theta} f(z)\right)^{\prime \prime \prime}}{z\left(\mathfrak{B}_{\eta, \lambda}^{\theta} f(z)\right)^{\prime}+\sigma z^{2}\left(\mathfrak{B}_{\eta, \lambda}^{\theta} f(z)\right)^{\prime \prime}} \\
= & 1+\mathcal{G}_{1}^{\delta}(t) u(z)+\mathcal{G}_{2}^{\delta}(t) u^{2}(z)+\cdots
\end{aligned}
$$

and

$$
\begin{aligned}
& \frac{w\left(\mathfrak{B}_{\eta, \lambda}^{\theta} g(w)\right)^{\prime}+(2 \sigma+1) w^{2}\left(\mathfrak{B}_{\eta, \lambda}^{\theta} g(w)\right)^{\prime \prime}+\sigma w^{3}\left(\mathfrak{B}_{\eta, \lambda}^{\theta} g(w)\right)^{\prime \prime \prime}}{w\left(\mathfrak{B}_{\eta, \lambda}^{\theta} g(w)\right)^{\prime}+\sigma w^{2}\left(\mathfrak{B}_{\eta, \lambda}^{\theta} g(w)\right)^{\prime \prime}} \\
= & 1+\mathcal{G}_{1}^{\delta}(t) v(w)+\mathcal{G}_{2}^{\delta}(t) v^{2}(w)+\cdots .
\end{aligned}
$$

where $u$ and $v$ have the forms (2.2) and (2.3). Combining (2.18) and (2.19), yield

$$
\begin{aligned}
& \frac{z\left(\mathfrak{B}_{\eta, \lambda}^{\theta} f(z)\right)^{\prime}+(2 \sigma+1) z^{2}\left(\mathfrak{B}_{\eta, \lambda}^{\theta} f(z)\right)^{\prime \prime}+\sigma z^{3}\left(\mathfrak{B}_{\eta, \lambda}^{\theta} f(z)\right)^{\prime \prime \prime}}{z\left(\mathfrak{B}_{\eta, \lambda}^{\theta} f(z)\right)^{\prime}+\sigma z^{2}\left(\mathfrak{B}_{\eta, \lambda}^{\theta} f(z)\right)^{\prime \prime}} \\
= & 1+\mathcal{G}_{1}^{\delta}(t) u_{1} z+\left[\mathcal{G}_{1}^{\delta}(t) u_{2}+\mathcal{G}_{2}^{\delta}(t) u_{1}^{2}\right] z^{2}+\cdots
\end{aligned}
$$

and

$$
\begin{gathered}
\frac{w\left(\mathfrak{B}_{\eta, \lambda}^{\theta} g(w)\right)^{\prime}+(2 \sigma+1) w^{2}\left(\mathfrak{B}_{\eta, \lambda}^{\theta} g(w)\right)^{\prime \prime}+\sigma w^{3}\left(\mathfrak{B}_{\eta, \lambda}^{\theta} g(w)\right)^{\prime \prime \prime}}{w\left(\mathfrak{B}_{\eta, \lambda}^{\theta} g(w)\right)^{\prime}+\sigma w^{2}\left(\mathfrak{B}_{\eta, \lambda}^{\theta} g(w)\right)^{\prime \prime}} \\
=1+\mathcal{G}_{1}^{\delta}(t) v_{1} w+\left[\mathcal{G}_{1}^{\delta}(t) v_{2}+\mathcal{G}_{2}^{\delta}(t) v_{1}^{2}\right] w^{2}+\cdots .
\end{gathered}
$$

Equating the coefficients in (2.20) and (2.21), we deduce that

$$
\frac{2 \theta(\sigma+1) \Gamma(\eta+\theta) \Gamma(\lambda+1) \Gamma(\eta+\lambda)}{\Gamma(\eta+\theta+\lambda+1) \Gamma(\eta) \Gamma(\lambda)} a_{2}=\mathcal{G}_{1}^{\delta}(t) u_{1},
$$

$$
\frac{3 \theta(\theta+1)(2 \sigma+1) \Gamma(\eta+\theta) \Gamma(\lambda+2) \Gamma(\eta+\lambda)}{\Gamma(\eta+\theta+\lambda+2) \Gamma(\eta) \Gamma(\lambda)} a_{3}
$$




$$
\begin{array}{r}
-\frac{4 \theta^{2}(\sigma+1)^{2} \Gamma^{2}(\eta+\theta) \Gamma^{2}(\lambda+1) \Gamma^{2}(\eta+\lambda)}{\Gamma^{2}(\eta+\theta+\lambda+1) \Gamma^{2}(\eta) \Gamma^{2}(\lambda)} a_{2}^{2}=\mathcal{G}_{1}^{\delta}(t) u_{2}+\mathcal{G}_{2}^{\delta}(t) u_{1}^{2}, \\
-\frac{2 \theta(\sigma+1) \Gamma(\eta+\theta) \Gamma(\lambda+1) \Gamma(\eta+\lambda)}{\Gamma(\eta+\theta+\lambda+1) \Gamma(\eta) \Gamma(\lambda)} a_{2}=\mathcal{G}_{1}^{\delta}(t) v_{1}
\end{array}
$$

and

$$
\begin{aligned}
& \frac{3 \theta(\theta+1)(2 \sigma+1) \Gamma(\eta+\theta) \Gamma(\lambda+2) \Gamma(\eta+\lambda)}{\Gamma(\eta+\theta+\lambda+2) \Gamma(\eta) \Gamma(\lambda)}\left(2 a_{2}^{2}-a_{3}\right) \\
& -\frac{4 \theta^{2}(\sigma+1)^{2} \Gamma^{2}(\eta+\theta) \Gamma^{2}(\lambda+1) \Gamma^{2}(\eta+\lambda)}{\Gamma^{2}(\eta+\theta+\lambda+1) \Gamma^{2}(\eta) \Gamma^{2}(\lambda)} a_{2}^{2}=\mathcal{G}_{1}^{\delta}(t) v_{2}+\mathcal{G}_{2}^{\delta}(t) v_{1}^{2} .
\end{aligned}
$$

In view of (2.22) and (2.24), we have

$$
u_{1}=-v_{1}
$$

and

$$
\frac{8 \theta^{2}(\sigma+1)^{2} \Gamma^{2}(\eta+\theta) \Gamma^{2}(\lambda+1) \Gamma^{2}(\eta+\lambda)}{\Gamma^{2}(\eta+\theta+\lambda+1) \Gamma^{2}(\eta) \Gamma^{2}(\lambda)} a_{2}^{2}=\left(G_{1}^{\delta}(t)\right)^{2}\left(u_{1}^{2}+v_{1}^{2}\right) .
$$

If we add (2.23) to (2.25), we conclude that

$$
\frac{2 \theta \Gamma(\eta+\theta) \Gamma(\eta+\lambda)}{\Gamma(\eta) \Gamma(\lambda)} \Upsilon(\sigma, \eta, \theta, \lambda) a_{2}^{2}=\mathcal{G}_{1}^{\delta}(t)\left(u_{2}+v_{2}\right)+\mathcal{G}_{2}^{\delta}(t)\left(u_{1}^{2}+v_{1}^{2}\right),
$$

where $\Upsilon(\sigma, \eta, \theta, \lambda)$ is given by (2.17).

By substitute the value of $u_{1}^{2}+v_{1}^{2}$ from (2.27) in (2.28), yields

$$
\begin{gathered}
{\left[\frac{2 \theta \Gamma(\eta+\theta) \Gamma(\eta+\lambda)}{\Gamma(\eta) \Gamma(\lambda)} \Upsilon(\sigma, \eta, \theta, \lambda)-\frac{8 \theta^{2}(\sigma+1)^{2} \Gamma^{2}(\eta+\theta) \Gamma^{2}(\lambda+1) \Gamma^{2}(\eta+\lambda) \mathcal{G}_{2}^{\delta}(t)}{\Gamma^{2}(\eta+\theta+\lambda+1) \Gamma^{2}(\eta) \Gamma^{2}(\lambda)\left(\mathcal{G}_{1}^{\delta}(t)\right)^{2}}\right] a_{2}^{2}} \\
=\mathcal{G}_{1}^{\delta}(t)\left(u_{2}+v_{2}\right),
\end{gathered}
$$

or equivalently

$$
a_{2}^{2}=\frac{\Gamma^{2}(\eta+\theta+\lambda+1) \Gamma^{2}(\eta) \Gamma^{2}(\lambda)\left(\mathcal{G}_{1}^{\delta}(t)\right)^{3}\left(u_{2}+v_{2}\right)}{2 \theta \Upsilon(\sigma, \eta, \theta, \lambda) \Gamma(\eta+\theta) \Gamma(\eta+\lambda) \Gamma^{2}(\eta+\theta+\lambda+1) \Gamma(\eta) \Gamma(\lambda)\left(\mathcal{G}_{1}^{\delta}(t)\right)^{2}},
$$

Further computations using (1.3), (2.7) and (2.29), we obtain 


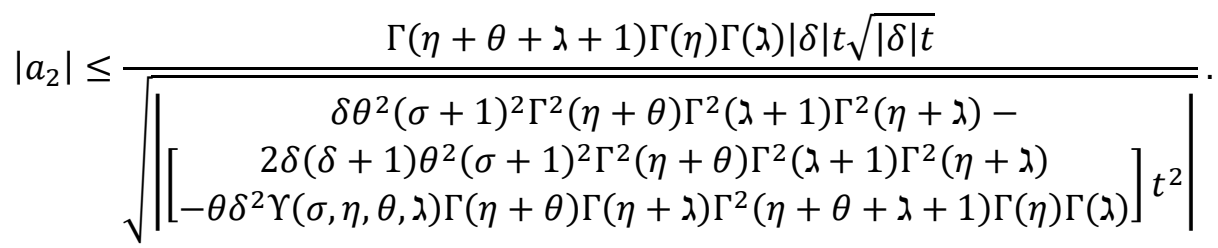

Next, if we subtract (2.25) from (2.23), we deduce that

$$
\begin{aligned}
& \frac{6 \theta(\theta+1)(2 \sigma+1) \Gamma(\eta+\theta) \Gamma(\lambda+2) \Gamma(\eta+\lambda)}{\Gamma(\eta+\theta+\lambda+2) \Gamma(\eta) \Gamma(\lambda)}\left(a_{3}-a_{2}^{2}\right) \\
= & \mathcal{G}_{1}^{\delta}(t)\left(u_{2}-v_{2}\right)+\mathcal{G}_{2}^{\delta}(t)\left(u_{1}^{2}-v_{1}^{2}\right) .
\end{aligned}
$$

In view of (2.26) and (2.27), we get from (2.30)

$$
\begin{aligned}
a_{3}=\frac{\Gamma^{2}(\eta+\theta+\lambda+1) \Gamma^{2}(\eta) \Gamma^{2}(\lambda)\left(\mathcal{G}_{1}^{\delta}(t)\right)^{2}}{8 \theta^{2}(\sigma+1)^{2} \Gamma^{2}(\eta+\theta) \Gamma^{2}(\lambda+1) \Gamma^{2}(\eta+\lambda)}\left(u_{1}^{2}+v_{1}^{2}\right) \\
\quad+\frac{\Gamma(\eta+\theta+\lambda+2) \Gamma(\eta) \Gamma(\lambda) \mathcal{G}_{1}^{\delta}(t)}{6 \theta(\theta+1)(2 \sigma+1) \Gamma(\eta+\theta) \Gamma(\lambda+2) \Gamma(\eta+\lambda)}\left(u_{2}-v_{2}\right) .
\end{aligned}
$$

Thus applying (1.3), we obtain

$$
\begin{aligned}
& \left|a_{3}\right| \leq \frac{\Gamma^{2}(\eta+\theta+\lambda+1) \Gamma^{2}(\eta) \Gamma^{2}(\lambda) \delta^{2} t^{2}}{\theta^{2}(\sigma+1)^{2} \Gamma^{2}(\eta+\theta) \Gamma^{2}(\lambda+1) \Gamma^{2}(\eta+\lambda)} \\
& +\frac{2 \Gamma(\eta+\theta+\lambda+2) \Gamma(\eta) \Gamma(\lambda)|\delta| t}{3 \theta(\theta+1)(2 \sigma+1) \Gamma(\eta+\theta) \Gamma(\lambda+2) \Gamma(\eta+\lambda)} .
\end{aligned}
$$

Putting $\sigma=0$ in Theorem 2.2, we demonstrate the next outcome:

Corollary 2.2. For $t \in\left(\frac{1}{2}, 1\right]$ and $\delta$ is a nonzero real constant, let $f \in \mathcal{A}$ be in the family $\mathfrak{H}_{\Sigma}(\eta, \theta, \lambda, t, \delta)$. Then

$$
\left|a_{2}\right| \leq \frac{\Gamma(\eta+\theta+\lambda+1) \Gamma(\eta) \Gamma(\lambda)|\delta| t \sqrt{|\delta| t}}{\sqrt{\delta \theta^{2} \Gamma^{2}(\eta+\theta) \Gamma^{2}(\lambda+1) \Gamma^{2}(\eta+\lambda)}}
$$

and

$$
\left|a_{3}\right| \leq \frac{\Gamma^{2}(\eta+\theta+\lambda+1) \Gamma^{2}(\eta) \Gamma^{2}(\lambda) \delta^{2} t^{2}}{\theta^{2} \Gamma^{2}(\eta+\theta) \Gamma^{2}(\lambda+1) \Gamma^{2}(\eta+\lambda)}+\frac{2 \Gamma(\eta+\theta+\lambda+2) \Gamma(\eta) \Gamma(\lambda)|\delta| t}{3 \theta(\theta+1) \Gamma(\eta+\theta) \Gamma(\lambda+2) \Gamma(\eta+\lambda)},
$$


where

$$
\mathfrak{A}(\eta, \theta, \lambda)=\frac{3(\theta+1) \Gamma(\lambda+2)}{\Gamma(\eta+\theta+\lambda+2)}-\frac{4 \theta \Gamma(\eta+\theta) \Gamma^{2}(\lambda+1) \Gamma(\eta+\lambda)}{\Gamma^{2}(\eta+\theta+\lambda+1) \Gamma(\eta) \Gamma(\lambda)} .
$$

Next theorems, show "Fekete-Szegö problem" of the families $\mathbb{T}_{\Sigma}(\gamma, \phi, \mu, \eta, \theta, \lambda, t, \delta)$ and $\mathbb{S}_{\Sigma}(\sigma, \eta, \theta, \lambda, t, \delta)$.

Theorem 2.3. For $0 \leq \gamma \leq 1,0 \leq \phi \leq 1,0 \leq \mu \leq 1, t \in\left(\frac{1}{2}, 1\right], \xi \in \mathbb{R}$ and $\delta$ is a nonzero real constant, let $f \in \mathcal{A}$ be in the family $\mathbb{T}_{\Sigma}(\gamma, \phi, \mu, \eta, \theta, \lambda, t, \delta)$. Then

$$
\begin{aligned}
& \left|a_{3}-\xi a_{2}^{2}\right| \\
& \int \frac{2 t|\delta| \Gamma(\eta+\theta+\lambda+2) \Gamma(\eta) \Gamma(\lambda)}{\theta(\theta+1)(\gamma+\phi(2 \mu+1)) \Gamma(\eta+\theta) \Gamma(\lambda+2) \Gamma(\eta+\lambda)} ; \\
& \text { for }|\xi-1| \leq \frac{\Gamma(\eta+\theta+\lambda+2) \Gamma(\eta) \Gamma(\lambda)}{2 \theta(\theta+1)(\gamma+\phi(2 \mu+1)) \Gamma(\eta+\theta) \Gamma(\lambda+2) \Gamma(\eta+\lambda)} \times \\
& \delta \theta^{2}(\gamma+\phi(\mu+1))^{2} \Gamma^{2}(\eta+\theta) \Gamma^{2}(\lambda+1) \Gamma^{2}(\eta+\lambda) \\
& \times\left|\frac{-2\left[\begin{array}{c}
\delta(\delta+1) \theta^{2}(\gamma+\phi(\mu+1))^{2} \Gamma^{2}(\eta+\theta) \Gamma^{2}(\lambda+1) \Gamma^{2}(\eta+\lambda) \\
-\theta \delta^{2} \Omega(\gamma, \phi, \mu, \eta, \theta, \lambda) \Gamma(\eta+\theta) \Gamma(\eta+\lambda) \Gamma^{2}(\eta+\theta+\lambda+1) \Gamma(\eta) \Gamma(\lambda)
\end{array}\right] t^{2}}{2 \delta^{2} t^{2} \Gamma^{2}(\eta+\theta+\lambda+1) \Gamma^{2}(\eta) \Gamma^{2}(\lambda)}\right| \\
& \leq\{ \\
& \frac{8 t^{3}\left|\delta^{3}\right| \Gamma^{2}(\eta+\theta+\lambda+1) \Gamma^{2}(\eta) \Gamma^{2}(\lambda)|\xi-1|}{\delta \theta^{2}(\gamma+\phi(\mu+1))^{2} \Gamma^{2}(\eta+\theta) \Gamma^{2}(\lambda+1) \Gamma^{2}(\eta+\lambda)} ; \\
& \text { for }|\xi-1| \geq \frac{\Gamma(\eta+\theta+\lambda+2) \Gamma(\eta) \Gamma(\lambda)}{2 \theta(\theta+1)(\gamma+\phi(2 \mu+1)) \Gamma(\eta+\theta) \Gamma(\lambda+2) \Gamma(\eta+\lambda)} \times
\end{aligned}
$$

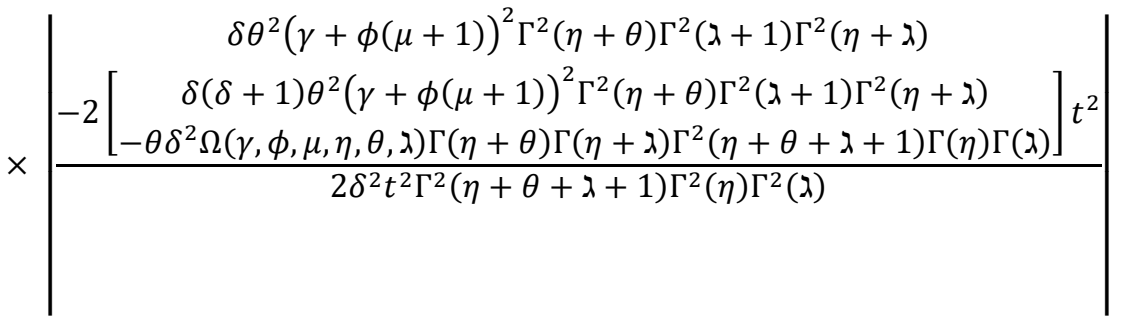

Proof. In the light of (2.15) and (2.16), we deduce that 


$$
\begin{gathered}
a_{3}-\xi a_{2}^{2}=(1-\xi) a_{2}^{2}+\frac{\Gamma(\eta+\theta+\lambda+2) \Gamma(\eta) \Gamma(\lambda) \mathcal{G}_{1}^{\delta}(t)\left(u_{2}-v_{2}\right)}{2 \theta(\theta+1)(\gamma+\phi(2 \mu+1)) \Gamma(\eta+\theta) \Gamma(\lambda+2) \Gamma(\eta+\lambda)} \\
=(1-\xi) \frac{\Gamma^{2}(\eta+\theta+\lambda+1) \Gamma^{2}(\eta) \Gamma^{2}(\lambda)\left(\mathcal{G}_{1}^{\delta}(t)\right)^{3}\left(u_{2}+v_{2}\right)}{\theta \Gamma(\eta+\theta) \Gamma(\eta+\lambda) \Gamma(\eta) \Gamma(\lambda) \Gamma^{2}(\eta+\theta+\lambda+1) \Omega(\gamma, \phi, \mu, \eta, \theta, \lambda)\left(\mathcal{G}_{1}^{\delta}(t)\right)^{2}-} \\
+\frac{2 \theta^{2}(\gamma+\phi(\mu+1))^{2} \Gamma^{2}(\eta+\theta) \Gamma^{2}(\lambda+1) \Gamma^{2}(\eta+\lambda) \mathcal{G}_{2}^{\delta}(t)}{2 \theta(\theta+1)(\gamma+\phi(2 \mu+1)) \Gamma(\eta+\theta) \Gamma(\lambda+2) \Gamma(\eta+\lambda)} \\
=\mathcal{G}_{1}^{\delta}(t)\left[\left(\psi(\xi)+\frac{\Gamma(\eta+\theta+\lambda+2) \Gamma(\eta) \Gamma(\lambda)}{2 \theta(\theta+1)(\gamma+\phi(2 \mu+1)) \Gamma(\eta+\theta) \Gamma(\lambda+2) \Gamma(\eta+\lambda)}\right) u_{2}\right. \\
\left.+\left(\psi(\xi)-\frac{\Gamma(\eta+\theta+\lambda+2) \Gamma(\eta) \Gamma(\lambda)}{2 \theta(\theta+1)(\gamma+\phi(2 \mu+1)) \Gamma(\eta+\theta) \Gamma(\lambda+2) \Gamma(\eta+\lambda)}\right) v_{2}\right],
\end{gathered}
$$

where

$$
\psi(\xi)=\frac{\Gamma^{2}(\eta+\theta+\lambda+1) \Gamma^{2}(\eta) \Gamma^{2}(\lambda)\left(\mathcal{G}_{1}^{\delta}(t)\right)^{2}(1-\xi)}{\theta \Gamma(\eta+\theta) \Gamma(\eta+\lambda) \Gamma(\eta) \Gamma(\lambda) \Gamma^{2}(\eta+\theta+\lambda+1) \Omega(\gamma, \phi, \mu, \eta, \theta, \lambda)\left(\mathcal{G}_{1}^{\delta}(t)\right)^{2}-} .
$$

According to (1.3), we deduce that

$$
\left|a_{3}-\xi a_{2}^{2}\right| \leq\left\{\begin{array}{l}
\frac{2 t|\delta| \Gamma(\eta+\theta+\lambda+2) \Gamma(\eta) \Gamma(\lambda)}{\theta(\theta+1)(\gamma+\phi(2 \mu+1)) \Gamma(\eta+\theta) \Gamma(\lambda+2) \Gamma(\eta+\lambda)} \\
0 \leq|\psi(\xi)| \leq \frac{\Gamma(\eta+\theta+\lambda+2) \Gamma(\eta) \Gamma(\lambda)}{2 \theta(\theta+1)(\gamma+\phi(2 \mu+1)) \Gamma(\eta+\theta) \Gamma(\lambda+2) \Gamma(\eta+\lambda)} \\
4 t|\delta||\psi(\xi)|, \\
|\psi(\xi)| \geq \frac{\Gamma(\eta+\theta+\lambda+2) \Gamma(\eta) \Gamma(\lambda)}{2 \theta(\theta+1)(\gamma+\phi(2 \mu+1)) \Gamma(\eta+\theta) \Gamma(\lambda+2) \Gamma(\eta+\lambda)}
\end{array}\right.
$$

After some computations, we obtain 
$\left|a_{3}-\xi a_{2}^{2}\right|$

$$
\begin{aligned}
& \int \frac{2 t|\delta| \Gamma(\eta+\theta+\lambda+2) \Gamma(\eta) \Gamma(\lambda)}{\theta(\theta+1)(\gamma+\phi(2 \mu+1)) \Gamma(\eta+\theta) \Gamma(\lambda+2) \Gamma(\eta+\lambda)} ; \\
& \text { for }|\xi-1| \leq \frac{\Gamma(\eta+\theta+\lambda+2) \Gamma(\eta) \Gamma(\lambda)}{2 \theta(\theta+1)(\gamma+\phi(2 \mu+1)) \Gamma(\eta+\theta) \Gamma(\lambda+2) \Gamma(\eta+\lambda)} \times \\
& \delta \theta^{2}(\gamma+\phi(\mu+1))^{2} \Gamma^{2}(\eta+\theta) \Gamma^{2}(\lambda+1) \Gamma^{2}(\eta+\lambda) \\
& \times\left|\frac{-2\left[\begin{array}{c}
\delta(\delta+1) \theta^{2}(\gamma+\phi(\mu+1))^{2} \Gamma^{2}(\eta+\theta) \Gamma^{2}(\lambda+1) \Gamma^{2}(\eta+\lambda) \\
-\theta \delta^{2} \Omega(\gamma, \phi, \mu, \eta, \theta, \lambda) \Gamma(\eta+\theta) \Gamma(\eta+\lambda) \Gamma^{2}(\eta+\theta+\lambda+1) \Gamma(\eta) \Gamma(\lambda)
\end{array}\right] t^{2}}{2 \delta^{2} t^{2} \Gamma^{2}(\eta+\theta+\lambda+1) \Gamma^{2}(\eta) \Gamma^{2}(\lambda)}\right| \\
& \leq\left\{\begin{array}{c}
8 t^{3}\left|\delta^{3}\right| \Gamma^{2}(\eta+\theta+\lambda+1) \Gamma^{2}(\eta) \Gamma^{2}(\lambda)|\xi-1| \\
\qquad \theta^{2}(\gamma+\phi(\mu+1))^{2} \Gamma^{2}(\eta+\theta) \Gamma^{2}(\lambda+1) \Gamma^{2}(\eta+\lambda) \\
-2\left[\begin{array}{c}
\delta(\delta+1) \theta^{2}(\gamma+\phi(\mu+1))^{2} \Gamma^{2}(\eta+\theta) \Gamma^{2}(\lambda+1) \Gamma^{2}(\eta+\lambda) \\
-\theta \delta^{2} \Omega(\gamma, \phi, \mu, \eta, \theta, \lambda) \Gamma(\eta+\theta) \Gamma(\eta+\lambda) \Gamma^{2}(\eta+\theta+\lambda+1) \Gamma(\eta) \Gamma(\lambda)
\end{array}\right]
\end{array} \mid\right. \\
& \text { for }|\xi-1| \geq \frac{\Gamma(\eta+\theta+\lambda+2) \Gamma(\eta) \Gamma(\lambda)}{2 \theta(\theta+1)(\gamma+\phi(2 \mu+1)) \Gamma(\eta+\theta) \Gamma(\lambda+2) \Gamma(\eta+\lambda)} \times \\
& \times\left|\begin{array}{c}
\delta \theta^{2}(\gamma+\phi(\mu+1))^{2} \Gamma^{2}(\eta+\theta) \Gamma^{2}(\lambda+1) \Gamma^{2}(\eta+\lambda) \\
-2\left[\begin{array}{c}
\delta(\delta+1) \theta^{2}(\gamma+\phi(\mu+1))^{2} \Gamma^{2}(\eta+\theta) \Gamma^{2}(\lambda+1) \Gamma^{2}(\eta+\lambda) \\
-\theta \delta^{2} \Omega(\gamma, \phi, \mu, \eta, \theta, \lambda) \Gamma(\eta+\theta) \Gamma(\eta+\lambda) \Gamma^{2}(\eta+\theta+\lambda+1) \Gamma(\eta) \Gamma(\lambda)
\end{array}\right] \\
\hline 2 \delta^{2} t^{2} \Gamma^{2}(\eta+\theta+\lambda+1) \Gamma^{2}(\eta) \Gamma^{2}(\lambda)
\end{array}\right|
\end{aligned}
$$

Putting $\xi=1$ in Theorem 2.3, we demonstrate the next outcome:

Corollary 2.3. For $0 \leq \gamma \leq 1,0 \leq \phi \leq 1,0 \leq \mu \leq 1, t \in\left(\frac{1}{2}, 1\right]$ and $\delta$ is a nonzero real constant, let $f \in \mathcal{A}$ be in the family $\mathbb{T}_{\Sigma}(\gamma, \phi, \mu, \eta, \theta, \lambda, t, \delta)$. Then

$$
\left|a_{3}-a_{2}^{2}\right| \leq \frac{2 t|\delta| \Gamma(\eta+\theta+\lambda+2) \Gamma(\eta) \Gamma(\lambda)}{\theta(\theta+1)(\gamma+\phi(2 \mu+1)) \Gamma(\eta+\theta) \Gamma(\lambda+2) \Gamma(\eta+\lambda)} .
$$

Putting $\phi=0$ and $\gamma=1$ in Theorem 2.3, we demonstrate the next outcome: 
Corollary 2.4. For $t \in\left(\frac{1}{2}, 1\right], \xi \in \mathbb{R}$ and $\delta$ is a nonzero real constant, let $f \in \mathcal{A}$ be in the family $\mathfrak{I}_{\Sigma}(\eta, \theta, \lambda, t, \delta)$. Then

$$
\begin{aligned}
& \left|a_{3}-\xi a_{2}^{2}\right| \\
& \int \frac{2 t|\delta| \Gamma(\eta+\theta+\lambda+2) \Gamma(\eta) \Gamma(\lambda)}{\theta(\theta+1) \Gamma(\eta+\theta) \Gamma(\lambda+2) \Gamma(\eta+\lambda)} ; \\
& \text { for }|\xi-1| \leq \frac{\Gamma(\eta+\theta+\lambda+2) \Gamma(\eta) \Gamma(\lambda)}{2 \theta(\theta+1) \Gamma(\eta+\theta) \Gamma(\lambda+2) \Gamma(\eta+\lambda)} \times \\
& \left.\times \mid \begin{array}{c}
\delta \theta^{2} \Gamma^{2}(\eta+\theta) \Gamma^{2}(\lambda+1) \Gamma^{2}(\eta+\lambda) \\
\delta(\delta+1) \theta^{2} \Gamma^{2}(\eta+\theta) \Gamma^{2}(\lambda+1) \Gamma^{2}(\eta+\lambda) \\
-2\left[\theta \delta^{2} \varsigma(\eta, \theta, \lambda) \Gamma(\eta+\theta) \Gamma(\eta+\lambda) \Gamma^{2}(\eta+\theta+\lambda+1) \Gamma(\eta) \Gamma(\lambda)\right.
\end{array}\right] t^{2} \mid
\end{aligned}
$$

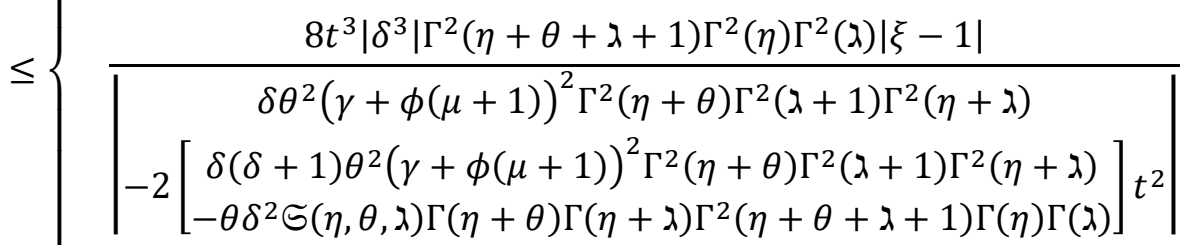

$$
\begin{aligned}
& \text { for }|\xi-1| \geq \frac{\Gamma(\eta+\theta+\lambda+2) \Gamma(\eta) \Gamma(\lambda)}{2 \theta(\theta+1)(\gamma+\phi(2 \mu+1)) \Gamma(\eta+\theta) \Gamma(\lambda+2) \Gamma(\eta+\lambda)} \times \\
& \times\left|\begin{array}{c}
\delta \theta^{2}(\gamma+\phi(\mu+1))^{2} \Gamma^{2}(\eta+\theta) \Gamma^{2}(\lambda+1) \Gamma^{2}(\eta+\lambda) \\
-2\left[\begin{array}{c}
\delta(\delta+1) \theta^{2}(\gamma+\phi(\mu+1))^{2} \Gamma^{2}(\eta+\theta) \Gamma^{2}(\lambda+1) \Gamma^{2}(\eta+\lambda) \\
-\theta \delta^{2} \subseteq(\eta, \theta, \lambda) \Gamma(\eta+\theta) \Gamma(\eta+\lambda) \Gamma^{2}(\eta+\theta+\lambda+1) \Gamma(\eta) \Gamma(\lambda)
\end{array}\right] t^{2} \\
2 \delta^{2} t^{2} \Gamma^{2}(\eta+\theta+\lambda+1) \Gamma^{2}(\eta) \Gamma^{2}(\lambda)
\end{array}\right|
\end{aligned}
$$

Theorem 2.4. For $0 \leq \sigma \leq 1, t \in\left(\frac{1}{2}, 1\right], \xi \in \mathbb{R}$ and $\delta$ is a nonzero real constant, let $f \in \mathcal{A}$ be in the family $\mathbb{S}_{\Sigma}(\sigma, \eta, \theta, \lambda, t, \delta)$. Then 
$\left|a_{3}-\xi a_{2}^{2}\right|$

$\int \frac{2 t|\delta| \Gamma(\eta+\theta+\lambda+2) \Gamma(\eta) \Gamma(\lambda)}{3 \theta(\theta+1)(2 \sigma+1) \Gamma(\eta+\theta) \Gamma(\lambda+2) \Gamma(\eta+\lambda)} ;$

for $|\xi-1| \leq \frac{\Gamma(\eta+\theta+\lambda+2) \Gamma(\eta) \Gamma(\lambda)}{3 \theta(\theta+1)(2 \sigma+1) \Gamma(\eta+\theta) \Gamma(\lambda+2) \Gamma(\eta+\lambda)} \times$

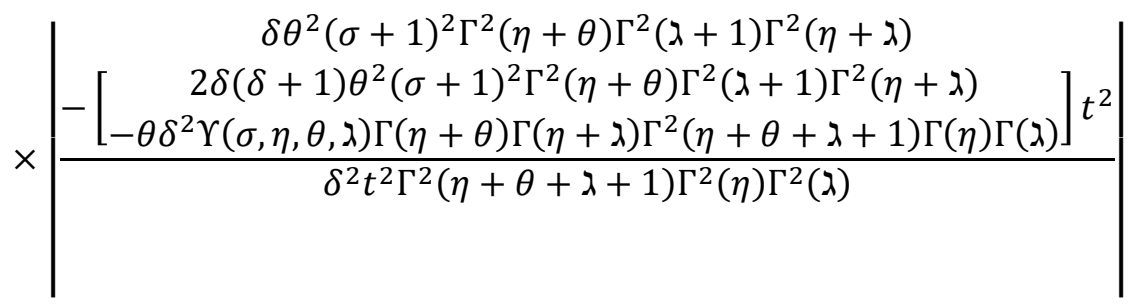

$\leq\left\{\begin{array}{c}2 t^{3}\left|\delta^{3}\right| \Gamma^{2}(\eta+\theta+\lambda+1) \Gamma^{2}(\eta) \Gamma^{2}(\lambda)|\xi-1| \\ \left.\qquad \begin{array}{c}\delta \theta^{2}(\sigma+1)^{2} \Gamma^{2}(\eta+\theta) \Gamma^{2}(\lambda+1) \Gamma^{2}(\eta+\lambda) \\ 2 \delta(\delta+1) \theta^{2}(\sigma+1)^{2} \Gamma^{2}(\eta+\theta) \Gamma^{2}(\lambda+1) \Gamma^{2}(\eta+\lambda) \\ -\theta \delta^{2} \Upsilon(\sigma, \eta, \theta, \lambda) \Gamma(\eta+\theta) \Gamma(\eta+\lambda) \Gamma^{2}(\eta+\theta+\lambda+1) \Gamma(\eta) \Gamma(\lambda)\end{array}\right] t^{2}\end{array}\right.$ for $|\xi-1| \geq \frac{\Gamma(\eta+\theta+\lambda+2) \Gamma(\eta) \Gamma(\lambda)}{3 \theta(\theta+1)(2 \sigma+1) \Gamma(\eta+\theta) \Gamma(\lambda+2) \Gamma(\eta+\lambda)} \times$

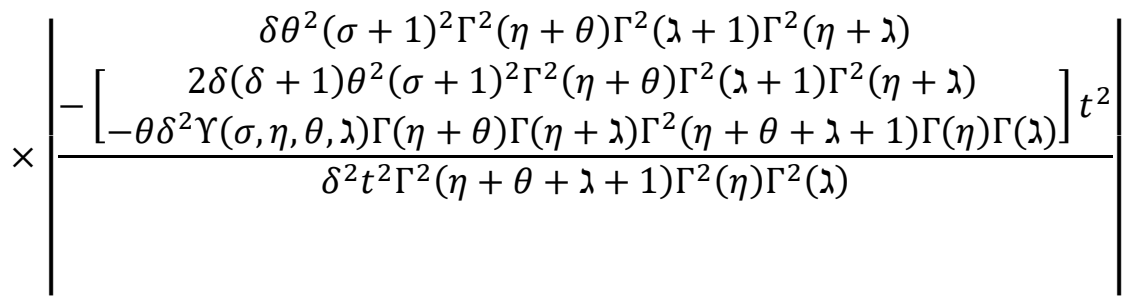

Proof. In view of (2.29) and (2.30), we deduce that

$$
\begin{gathered}
a_{3}-\xi a_{2}^{2}=(1-\xi) a_{2}^{2}+\frac{\Gamma(\eta+\theta+\lambda+2) \Gamma(\eta) \Gamma(\lambda) \mathcal{G}_{1}^{\delta}(t)\left(u_{2}-v_{2}\right)}{6 \theta(\theta+1)(2 \sigma+1) \Gamma(\eta+\theta) \Gamma(\lambda+2) \Gamma(\eta+\lambda)} \\
=(1-\xi) \frac{\Gamma^{2}(\eta+\theta+\lambda+1) \Gamma^{2}(\eta) \Gamma^{2}(\lambda)\left(\mathcal{G}_{1}^{\delta}(t)\right)^{3}\left(u_{2}+v_{2}\right)}{2 \theta \Upsilon(\sigma, \eta, \theta, \lambda) \Gamma(\eta+\theta) \Gamma(\eta+\lambda) \Gamma^{2}(\eta+\theta+\lambda+1) \Gamma(\eta) \Gamma(\lambda)\left(\mathcal{G}_{1}^{\delta}(t)\right)^{2}} \\
-8 \theta^{2}(\sigma+1)^{2} \Gamma^{2}(\eta+\theta) \Gamma^{2}(\lambda+1) \Gamma^{2}(\eta+\lambda) \mathcal{G}_{2}^{\delta}(t) \\
+\frac{\Gamma(\eta+\theta+\lambda+2) \Gamma(\eta) \Gamma(\lambda) \mathcal{G}_{1}^{\delta}(t)\left(u_{2}-v_{2}\right)}{6 \theta(\theta+1)(2 \sigma+1) \Gamma(\eta+\theta) \Gamma(\lambda+2) \Gamma(\eta+\lambda)}
\end{gathered}
$$




$$
\begin{aligned}
=\frac{\mathcal{G}_{1}^{\delta}(t)}{2}[(\varphi(\xi) & \left.+\frac{\Gamma(\eta+\theta+\lambda+2) \Gamma(\eta) \Gamma(\lambda)}{3 \theta(\theta+1)(2 \sigma+1) \Gamma(\eta+\theta) \Gamma(\lambda+2) \Gamma(\eta+\lambda)}\right) u_{2} \\
& \left.+\left(\varphi(\xi)-\frac{\Gamma(\eta+\theta+\lambda+2) \Gamma(\eta) \Gamma(\lambda)}{3 \theta(\theta+1)(2 \sigma+1) \Gamma(\eta+\theta) \Gamma(\lambda+2) \Gamma(\eta+\lambda)}\right) v_{2}\right]
\end{aligned}
$$

where

$$
\varphi(\xi)=\frac{\Gamma^{2}(\eta+\theta+\lambda+1) \Gamma^{2}(\eta) \Gamma^{2}(\lambda)\left(\mathcal{G}_{1}^{\delta}(t)\right)^{2}(1-\xi)}{\theta \Upsilon(\sigma, \eta, \theta, \lambda) \Gamma(\eta+\theta) \Gamma(\eta+\lambda) \Gamma^{2}(\eta+\theta+\lambda+1) \Gamma(\eta) \Gamma(\lambda)\left(\mathcal{G}_{1}^{\delta}(t)\right)^{2}} .
$$

According to (1.3), we deduce that

$$
\left|a_{3}-\xi a_{2}^{2}\right| \leq\left\{\begin{array}{l}
\frac{2 t|\delta| \Gamma(\eta+\theta+\lambda+2) \Gamma(\eta) \Gamma(\lambda)}{3 \theta(\theta+1)(2 \sigma+1) \Gamma(\eta+\theta) \Gamma(\lambda+2) \Gamma(\eta+\lambda)} \\
0 \leq|\varphi(\xi)| \leq \frac{\Gamma(\eta+\theta+\lambda+2) \Gamma(\eta) \Gamma(\lambda)}{3 \theta(\theta+1)(2 \sigma+1) \Gamma(\eta+\theta) \Gamma(\lambda+2) \Gamma(\eta+\lambda)} \\
2 t|\delta||\varphi(\xi)|, \\
|\varphi(\xi)| \geq \frac{\Gamma(\eta+\theta+\lambda+2) \Gamma(\eta) \Gamma(\lambda)}{3 \theta(\theta+1)(2 \sigma+1) \Gamma(\eta+\theta) \Gamma(\lambda+2) \Gamma(\eta+\lambda)}
\end{array} .\right.
$$

After some computations, we obtain 
$\left|a_{3}-\xi a_{2}^{2}\right|$

$\int \frac{2 t|\delta| \Gamma(\eta+\theta+\lambda+2) \Gamma(\eta) \Gamma(\lambda)}{3 \theta(\theta+1)(2 \sigma+1) \Gamma(\eta+\theta) \Gamma(\lambda+2) \Gamma(\eta+\lambda)}$

for $|\xi-1| \leq \frac{\Gamma(\eta+\theta+\lambda+2) \Gamma(\eta) \Gamma(\lambda)}{3 \theta(\theta+1)(2 \sigma+1) \Gamma(\eta+\theta) \Gamma(\lambda+2) \Gamma(\eta+\lambda)} \times$

$\times\left|\begin{array}{c}\delta \theta^{2}(\sigma+1)^{2} \Gamma^{2}(\eta+\theta) \Gamma^{2}(\lambda+1) \Gamma^{2}(\eta+\lambda) \\ -\left[\begin{array}{c}2 \delta(\delta+1) \theta^{2}(\sigma+1)^{2} \Gamma^{2}(\eta+\theta) \Gamma^{2}(\lambda+1) \Gamma^{2}(\eta+\lambda) \\ -\theta \delta^{2} \Upsilon(\sigma, \eta, \theta, \lambda) \Gamma(\eta+\theta) \Gamma(\eta+\lambda) \Gamma^{2}(\eta+\theta+\lambda+1) \Gamma(\eta) \Gamma(\lambda)\end{array}\right] t^{2} \\ \hline \delta^{2} t^{2} \Gamma^{2}(\eta+\theta+\lambda+1) \Gamma^{2}(\eta) \Gamma^{2}(\lambda)\end{array}\right|$

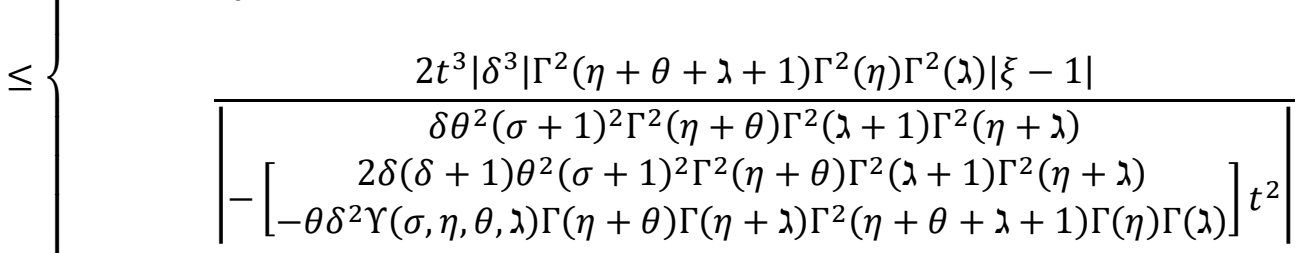

for $|\xi-1| \geq \frac{\Gamma(\eta+\theta+\lambda+2) \Gamma(\eta) \Gamma(\lambda)}{3 \theta(\theta+1)(2 \sigma+1) \Gamma(\eta+\theta) \Gamma(\lambda+2) \Gamma(\eta+\lambda)} \times$

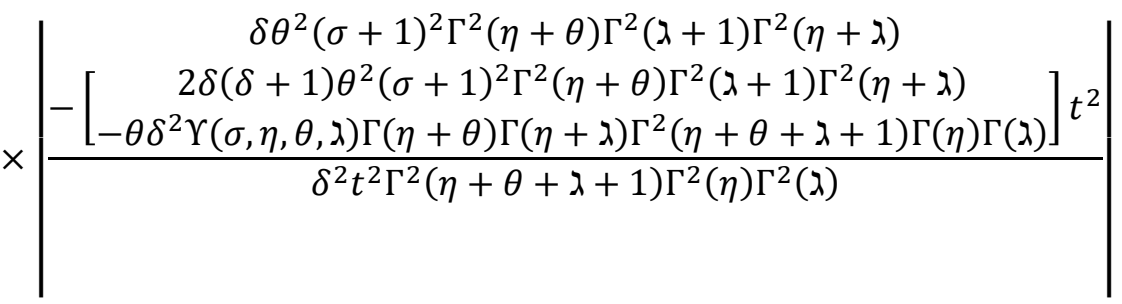

Putting $\xi=1$ in Theorem 2.4, we demonstrate the next outcome:

Corollary 2.5. For $0 \leq \sigma \leq 1, t \in\left(\frac{1}{2}, 1\right]$ and $\delta$ is a nonzero real constant, let $f \in \mathcal{A}$ be in the family $\mathbb{S}_{\Sigma}(\sigma, \eta, \theta, \lambda, t, \delta)$. Then

$$
\left|a_{3}-a_{2}^{2}\right| \leq \frac{2 t|\delta| \Gamma(\eta+\theta+\lambda+2) \Gamma(\eta) \Gamma(\lambda)}{3 \theta(\theta+1)(2 \sigma+1) \Gamma(\eta+\theta) \Gamma(\lambda+2) \Gamma(\eta+\lambda)} .
$$

Putting $\sigma=0$ in Theorem 2.4, we demonstrate the next outcome:

Corollary 2.6. For $t \in\left(\frac{1}{2}, 1\right], \xi \in \mathbb{R}$ and $\delta$ is a nonzero real constant, let $f \in \mathcal{A}$ be in the family $\mathfrak{G}_{\Sigma}(\eta, \theta, \lambda, t, \delta)$. Then 


$$
\begin{aligned}
& \left|a_{3}-\xi a_{2}^{2}\right| \\
& \int \frac{2 t|\delta| \Gamma(\eta+\theta+\lambda+2) \Gamma(\eta) \Gamma(\lambda)}{3 \theta(\theta+1) \Gamma(\eta+\theta) \Gamma(\lambda+2) \Gamma(\eta+\lambda)} ; \\
& \text { for }|\xi-1| \leq \frac{\Gamma(\eta+\theta+\lambda+2) \Gamma(\eta) \Gamma(\lambda)}{3 \theta(\theta+1) \Gamma(\eta+\theta) \Gamma(\lambda+2) \Gamma(\eta+\lambda)} \times \\
& \left.\times \mid \begin{array}{c}
\delta \theta^{2} \Gamma^{2}(\eta+\theta) \Gamma^{2}(\lambda+1) \Gamma^{2}(\eta+\lambda) \\
2 \delta(\delta+1) \theta^{2} \Gamma^{2}(\eta+\theta) \Gamma^{2}(\lambda+1) \Gamma^{2}(\eta+\lambda) \\
-\theta \delta^{2} \mathfrak{A}(\eta, \theta, \lambda) \Gamma(\eta+\theta) \Gamma(\eta+\lambda) \Gamma^{2}(\eta+\theta+\lambda+1) \Gamma(\eta) \Gamma(\lambda)
\end{array}\right] t^{2} \mid
\end{aligned}
$$

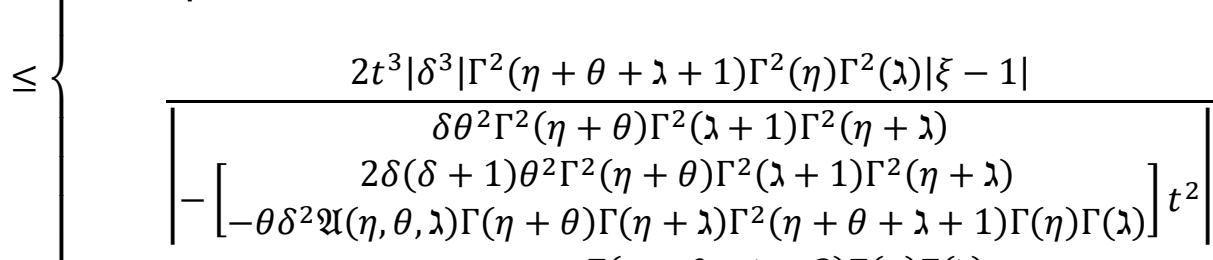

$$
\begin{aligned}
& \text { for }|\xi-1| \geq \frac{\Gamma(\eta+\theta+\lambda+2) \Gamma(\eta) \Gamma(\lambda)}{3 \theta(\theta+1) \Gamma(\eta+\theta) \Gamma(\lambda+2) \Gamma(\eta+\lambda)} \times \\
& \left.\times \mid \begin{array}{c}
\delta \theta^{2} \Gamma^{2}(\eta+\theta) \Gamma^{2}(\lambda+1) \Gamma^{2}(\eta+\lambda) \\
2 \delta(\delta+1) \theta^{2} \Gamma^{2}(\eta+\theta) \Gamma^{2}(\lambda+1) \Gamma^{2}(\eta+\lambda) \\
-\theta \delta^{2} \mathfrak{A}(\eta, \theta, \lambda) \Gamma(\eta+\theta) \Gamma(\eta+\lambda) \Gamma^{2}(\eta+\theta+\lambda+1) \Gamma(\eta) \Gamma(\lambda)
\end{array}\right] t^{2} \mid
\end{aligned}
$$

\section{Conclusion}

The primary objective was to create the families $\mathbb{T}_{\Sigma}(\gamma, \phi, \mu, \eta, \theta, \lambda, t, \delta)$ and $\mathbb{S}_{\Sigma}(\sigma, \eta, \theta, \lambda, t, \delta)$ of bi-univalent functions which governed by Gegenbauer polynomials. We generated Taylor coefficient inequalities for functions in the families $\mathbb{T}_{\Sigma}(\gamma, \phi, \mu, \eta, \theta, \lambda, t, \delta)$ and $\mathbb{S}_{\Sigma}(\sigma, \eta, \theta, \lambda, t, \delta)$ and viewed the famous Fekete-Szegö problem.

\section{References}

[1] E. A. Adegani, S. Bulut and A. A. Zireh, Coefficient estimates for a subclass of analytic bi-univalent functions, Bull. Korean Math. Soc. 55(2) (2018), 405-413. 
[2] A. G. Alamoush, On subclass of analytic bi-close-to-convex functions, Int. J. Open Problems Complex Analysis 13 (2021), 10-18.

[3] A. Amourah, A. Alamoush and M. Al-Kaseasbeh, Gegenbauer polynomials and biunivalent functions, Palestine Journal of Mathematics 10(2) (2021), 625-632.

[4] S. Altinkaya and S. Yalçin, On a new subclass of bi-univalent functions of Sakaguchi type satisfying subordinate conditions, Malaya Journal of Mathematics 5(2) (2017), 305309.

[5] S. Altinkaya and S. Yalçin, On the Chebyshev polynomial coefficient problem of some subclasses of bi-univalent functions, Gulf Journal of Mathematics 5(3) (2017), 34-40.

[6] S. Altinkaya and S. Yalçin, Poisson distribution series for certain subclasses of starlike functions with negative coefficients, Annals of Oradea University Mathematics Fascicola 24(2) (2017), 5-8.

[7] H. Bateman, Higher Transcendental Functions, McGraw-Hill, 1953.

[8] S. Bulut, Faber polynomial coefficient estimates for a subclass of analytic bi-univalent functions, Filomat 30(6) (2016), 1567-1575. https://doi.org/10.2298/FIL1606567B

[9] S. Bulut and A. K. Wanas, Coefficient estimates for families of bi-univalent functions defined by Ruscheweyh derivative operator, Mathematica Moravica 25(1) (2021), 71-80. https://doi.org/10.5937/MatMor2101071B

[10] M. Caglar, E. Deniz, H. M. Srivastava, Second Hankel determinant for certain subclasses of bi-univalent functions, Turk. J. Math. 41 (2017), 694-706.

https://doi.org/10.3906/mat-1602-25

[11] E. Deniz, Certain subclasses of bi-univalent functions satisfying subordinate conditions, J. Class. Anal. 2 (2013), 49-60. https://doi.org/10.7153/jca-02-05

[12] B. Doman, The Classical Orthogonal Polynomials, World Scientific, 2015. https://doi.org/10.1142/9700

[13] P. L. Duren, Univalent Functions, Grundlehren der Mathematischen Wissenschaften, Band 259, Springer Verlag, New York, Berlin, Heidelberg and Tokyo, 1983.

[14] S. S. Eker, Coefficient bounds for subclasses of $m$-fold symmetric bi-univalent functions, Turk. J. Math. 40(2016), 641-646. https://doi.org/10.3906/mat-1503-58

[15] S. M. El-Deeb, T. Bulboaca and J. Dziok, Pascal distribution series connected with certain subclasses of univalent functions, Kyungpook Math. J. 59(2) (2019), 301-314.

[16] M. Fekete and G. Szegö, Eine bemerkung uber ungerade schlichte funktionen, J. Lond. Math. Soc. 2 (1933), 85-89. https://doi.org/10.1112/jlms/s1-8.2.85 
[17] B. A. Frasin, Coefficient bounds for certain classes of bi-univalent functions, Hacettepe Journal of Mathematics and Statistics 43(3) (2014), 383-389.

[18] H. O. Guney, G. Murugusundaramoorthy and J. Sokol, Subclasses of bi-univalent functions related to shell-like curves connected with Fibonacci numbers, Acta Univ. Sapient. Math. 10 (2018), 70-84. https://doi.org/10.2478/ausm-2018-0006

[19] K. Kiepiela, I. Naraniecka, and J. Szynal, The Gegenbauer polynomials and typically real functions, Journal of Computational and Applied Mathematics 153(1-2) (2003), 273-28. https://doi.org/10.1016/S0377-0427(02)00642-8

[20] A. Legendre, Recherches sur l'attraction des spheroids homogénes, vol. 10, Mémoires présentes par divers savans à l'Académie des Sciences de l'Institut de France, Paris, 1785.

[21] N. Magesh and S. Bulut, Chebyshev polynomial coefficient estimates for a class of analytic bi-univalent functions related to pseudo-starlike functions, Afr. Mat. 29(1-2) (2018), 203-209. https://doi.org/10.1007/s13370-017-0535-3

[22] N. Magesh and J. Yamini, Fekete-Szegö problem and second Hankel determinant for a class of bi-univalent functions, Tbilisi Math. J. 11(1) (2018), 141-157.

https://doi.org/10.32513/tbilisi/1524276036

[23] S. S. Miller and P. T. Mocanu, Differential Subordinations: Theory and Applications, Series on Monographs and Textbooks in Pure and Applied Mathematics Vol. 225, Marcel Dekker Inc., New York and Basel, 2000.

[24] W. Nazeer, Q. Mehmood, S. M. Kang and A. U. Haq, An application of Binomial distribution series on certain analytic functions, Journal of Computational Analysis and Applications 26 (2019), 11-17.

[25] A. O. Pall-Szabo and A. K. Wanas, Coefficient estimates for some new classes of biBazilevic functions of Ma-Minda type involving the Salagean integro-differential operator, Quaestiones Mathematicae 44 (4) (2021), 495-502.

https://doi.org/10.2989/16073606.2020.1727581

[26] S. Porwal and M. Kumar, A unified study on starlike and convex functions associated with Poisson distribution series, Afr. Mat. 27(2016), 10-21.

https://doi.org/10.1007/s13370-016-0398-z

[27] M. Reimer, Multivariate Polynomial Approximation, Birkhäuser, 2012.

[28] B. Seker, On a new subclass of bi-univalent functions defined by using Salagean operator, Turk. J. Math. 42 (2018), 2891-2896. https://doi.org/10.3906/mat-1507-100 
[29] B. Seker and I. Taymur, On subclasses of $m$-fold symmetric bi-univalent functions, TWMS J. App. and Eng. Math. 11(2) (2021), 598-604.

[30] T. G. Shaba and A. B. Patil, Coefficient estimates for certain subclasses of $m$-fold symmetric bi-univalent functions associated with pseudo-starlike functions, Earthline Journal of Mathematical Sciences 6(2) (2021), 209-223. https://doi.org/10.34198/ejms.6221.209223

[31] T. G. Shaba, On some subclasses of bi-pseudo-starlike functions defined by Salagean differential operator, Asia Pac. J. Math. 8(6) (2021), 1-11. https://doi.org/10.28924/APJM/8-6

[32] H. M. Srivastava, Operators of basic (or q-) calculus and fractional q-calculus and their applications in geometric function theory of complex analysis, Iran. J. Sci. Technol. Trans. A: Sci. 44 (2020), 327-344. https://doi.org/10.1007/s40995-019-00815-0

[33] H. M. Srivastava, S. Altinkaya and S. Yalçin, Certain subclasses of bi-univalent functions associated with the Horadam polynomials, Iran. J. Sci. Technol. Trans. A: Sci. 43 (2019), 1873-1879. https://doi.org/10.1007/s40995-018-0647-0

[34] H. M. Srivastava, S. S. Eker, S. G. Hamidi and J. M. Jahangiri, Faber polynomial coefficient estimates for bi-univalent functions defined by the Tremblay fractional derivative operator, Bull. Iran. Math. Soc. 44 (2018), 149-157. https://doi.org/10.1007/s41980-018-0011-3

[35] H. M. Srivastava, S. Hussain, A. Raziq and M. Raza, The Fekete-Szegö functional for a subclass of analytic functions associated with quasi-subordination, Carpathian J. Math. 34 (2018), 103-113. https://doi.org/10.37193/CJM.2018.01.11

[36] H. M. Srivastava, A. K. Mishra and P. Gochhayat, Certain subclasses of analytic and biunivalent functions, Appl. Math. Lett. 23 (2010), 1188-1192. https://doi.org/10.1016/j.aml.2010.05.009

[37] H. M. Srivastava and A. K. Wanas, Initial Maclaurin coefficient bounds for new subclasses of analytic and $m$-fold symmetric bi-univalent functions defined by a linear combination, Kyungpook Math. J. 59(3) (2019), 493-503.

[38] H. M. Srivastava, A. K. Wanas and G. Murugusundaramoorthy, Certain family of biunivalent functions associated with Pascal distribution series based on Horadam polynomials, Surveys in Mathematics and its Applications 16 (2021), 193-205.

[39] H. M. Srivastava, A. K. Wanas and R. Srivastava, Applications of the q-Srivastava-Attiya operator involving a certain family of bi-univalent functions associated with the Horadam polynomials, Symmetry 13 (2021), Art. ID 1230, pp. 1-14.

https://doi.org/10.3390/sym13071230 
[40] S. R. Swamy, A. K. Wanas and Y. Sailaja, Some special families of holomorphic and Salagean type bi-univalent functions associated with $(m, n)$-Lucas polynomials, Communications in Mathematics and Applications 11(4) (2020), 563-574. https://doi.org/10.15672/hujms.695858

[41] A. K. Wanas, Horadam polynomials for a new family of $\lambda$-pseudo bi-univalent functions associated with Sakaguchi type functions, Afr. Mat. 32 (2021), 879-889. https://doi.org/10.1007/s13370-020-00867-1

[42] A. K. Wanas and N. A. Al-Ziadi, Applications of Beta negative binomial distribution series on holomorphic functions, Earthline Journal of Mathematical Sciences 6(2) (2021), 271-292. https://doi.org/10.34198/ejms.6221.271292

[43] A. K. Wanas and J. A. Khuttar, Applications of Borel distribution series on analytic functions, Earthline Journal of Mathematical Sciences 4(1) (2020), 71-82. https://doi.org/10.34198/ejms.4120.7182

This is an open access article distributed under the terms of the Creative Commons Attribution License (http://creativecommons.org/licenses/by/4.0/), which permits unrestricted, use, distribution and reproduction in any medium, or format for any purpose, even commercially provided the work is properly cited. 